\title{
Role of weak measurements on states ordering and monogamy of quantum correlation
}

\author{
Ming-Liang $\mathrm{Hu},{ }^{1,2,-\circledast}$ Heng Fan, ${ }^{3}$ and Dong-Ping Tian ${ }^{1}$ \\ ${ }^{1}$ School of Science, Xi'an Jiaotong University, Xi'an 710049, China \\ ${ }^{2}$ School of Science, Xi'an University of Posts and Telecommunications, Xi'an 710121, China \\ ${ }^{3}$ Beijing National Laboratory for Condensed Matter Physics, \\ Institute of Physics, Chinese Academy of Sciences, Beijing 100190, China
}

\begin{abstract}
The information-theoretic definition of quantum correlation, e.g., quantum discord, is measurement dependent. By considering the more general quantum measurements, weak measurements, which include the projective measurement as a limiting case, we show that while weak measurements can enable one to capture more quantumness of correlation in a state, it can also induce other counterintuitive quantum effects. Specifically, we show that the general measurements with different strengths can impose different orderings for quantum correlations of some states. It can also modify the monogamous character for certain classes of states as well which may diminish the usefulness of quantum correlation as a resource in some protocols. In this sense, we say that the weak measurements play a dual role in defining quantum correlation.

PACS numbers: 03.65.Ud, 03.65.Ta, 03.67.Mn

Key Words: Quantum discord; Weak measurement; Monogamy
\end{abstract}

\section{INTRODUCTION}

Quantum correlation plays a crucial role in many quantum computation and information processing tasks [1]. Quantum discord (QD) [2-5], which goes beyond the traditional measure of quantum correlation, i.e., quantum entanglement, is proposed to be responsible for the power of a mixed state quantum algorithm with vanishing or negligible entanglement [6]. It has potential applications in detecting critical points of quantum phase transitions even at finite temperatures [79]. QD is also found to be the necessary resource for remote state preparation [10], quantum state discrimination [11, 12], and quantum locking [13, 14]. A connection between QD consumption and the quantum advantage for encoding information has been identified as well [15]. These findings have prompted a huge surge of interest in understanding QD from different perspectives, such as its operational interpretation via quantum state merging [16, 17] and teleportation fidelity [18], the generation of QD via local operations [19-23], the discording power of nonlocal unitary gates [24], and other related issues of QD [25-29]; see a recent review paper [30] for more results.

Due to the fundamental significance and potential applications, various measures of QD [2-5], as well as other related measures of quantum correlations [31-36], have been introduced. The general positive operator valued measurements (POVMs) are proposed in the original definitions of these measures. On the other hand, in view of the generally negligible improvement by doing minimization over full POVMs [37, 38], measures of quantum correlation are usually evaluated by restricting to only projective measurements. The process of projective measurements are performed by constructing a set of orthogonal projectors in the Hilbert space of a Hermitian operator $\mathcal{A}$, and the possible outcomes of the measurements are given by the spectra of $\mathcal{A}$. This process usu-

*Electronic address: mingliang0301@163.com ally induces strong perturbations to the measured system, and possibly constrains one's ability to extract as much quantum correlations as possible.

Weak measurement can provides new insights into the study of some fundamental problems of quantum mechanics and has already been realized experimentally [39-41]. Also it can be used for signal amplification practically and for state tomography [42]. Particularly, these measurement processes are universal in that any generalized measurements can be decomposed into a sequence of weak measurements [43]. Since its fundamental role in quantum theory and practical applications, it is natural to consider the quantumness of correlations by weak measurement. The weak measurement which can be implemented by coupling the system to the measurement apparatus weakly and generally have small influence on a quantum state due to the partial collapsing of the measured wavefunction [43-47]. This differs it from projective measurement performed in standard QD. The quantum correlation based on weak measurements is proposed as super quantum discord [48]. This new quantifier has been shown to play a potential role in the protocol of optimal assisted state discrimination where entanglement is totally not necessary [49], and it has also stimulated other related definition of quantum correlations [50].

The super discord is always larger than the normal discord defined by the strong (projective) measurements [48], and this may be regarded as a figure of merit by using the weak measurements in characterizing quantumness of correlations in a state. But just as every coin has two sides, here we will show that the use of weak measurements in defining quantum correlations can also induce other counterintuitive effects. As explicit examples, we will show that the super discord captured by the weak measurements with different strengths can impose different orderings of quantum states. This phenomenon is very different from those of the states ordering obtained in the literature [51-55], which are easy to understand as they are induced by different correlation measures, e.g., the entropic measure of discord [2] and the geometric measure of discord [4]. Moreover, we will also show that the super discord can 
change the monogamy nature for certain classes of states $[56-$ 63]. Detailed examples show that this change presents in a wide class of quantum states, and therefore may result in failure of certain quantum tasks, such as the protocol that distinguishes the generalized Greenberger-Horne-Zeilinger (GHZ) states from the generalized $W$ states by using the monogamy conditions of QD [57].

\section{DEFINITION OF SUPER DISCORD}

In this section, we will introduce the concept of super discord [48]. Its definition is somewhat similar as that of the normal discord introduced by Ollivier and Zurek [2]. The only difference is that the original projective operators are replaced by the weak measurement operators of the following form [43]

$$
\begin{aligned}
& \mathcal{P}_{+}(x)=\sqrt{\frac{1-\tanh x}{2}} \Pi_{0}+\sqrt{\frac{1+\tanh x}{2}} \Pi_{1}, \\
& \mathcal{P}_{-}(x)=\sqrt{\frac{1+\tanh x}{2}} \Pi_{0}+\sqrt{\frac{1-\tanh x}{2}} \Pi_{1},
\end{aligned}
$$

where the strength of the measurement process is parameterized by a parameter $x \in \mathbb{R} . \Pi_{0}$ and $\Pi_{1}$ are the orthogonal projectors summing to the identity, and therefore $\mathcal{P}_{+}^{2}+\mathcal{P}_{-}^{2}=I$. Along this line of measurement formalism, one can then obtain the nonselective postmeasurement state as

$$
\rho_{A \mid \mathcal{P}_{ \pm}^{B}}=\frac{\operatorname{Tr}_{B}\left[\left(I \otimes \mathcal{P}_{ \pm}^{B}\right) \rho_{A B}\left(I \otimes \mathcal{P}_{ \pm}^{B}\right)\right]}{p_{ \pm}}
$$

after the weak measurements being performed on party $B$, and $p_{ \pm}=\operatorname{Tr}\left[\left(I \otimes \mathcal{P}_{ \pm}^{B}\right) \rho_{A B}\left(I \otimes \mathcal{P}_{ \pm}^{B}\right)\right]$ is the probability distribution for the measurement outcomes. Then the super discord is defined as

$$
D_{w}^{\leftarrow}\left(\rho_{A B}\right)=\min _{\left\{\Pi_{k}^{B}\right\}} S_{w}\left(A \mid\left\{\mathcal{P}_{ \pm}^{B}\right\}\right)-S(A \mid B)
$$

where the minimization is taken over the complete set of the projection-valued measurements $\left\{\Pi_{k}^{B}\right\}$. The conditional von Neumann entropy for the premeasurement state $\rho_{A B}$ is denoted by $S(A \mid B)=S\left(\rho_{A B}\right)-S\left(\rho_{B}\right)$, while the averaged conditional von Neumann entropy for the postmeasurement state is denoted by

$$
S_{w}\left(A \mid\left\{\mathcal{P}_{ \pm}^{B}\right\}\right)=p_{+} S\left(\rho_{A \mid \mathcal{P}_{+}^{B}}\right)+p_{-} S\left(\rho_{A \mid \mathcal{P}_{-}^{B}}\right),
$$

with $\rho_{B(A)}=\operatorname{Tr}_{A(B)} \rho_{A B}$ being the reduced density operator of $\rho_{A B}$, and $S(\rho)=-\operatorname{Tr}\left(\rho \log _{2} \rho\right)$ represents the von Neumann entropy [64].

\section{STATES ORDERING WITH SUPER DISCORD}

Given two quantum correlation measures, $\mathcal{Q}$ and $\mathcal{R}$, they are said to give the unique states ordering if and only if the following condition

$$
\mathcal{Q}\left(\rho_{1}\right) \geqslant \mathcal{Q}\left(\rho_{2}\right) \Longleftrightarrow \mathcal{R}\left(\rho_{1}\right) \geqslant \mathcal{R}\left(\rho_{2}\right)
$$

is satisfied for arbitrary $\rho_{1}$ and $\rho_{2}$.

For the entanglement measures of the concurrence [65] and the negativity [66], or quantum correlation measures of the entropic discord [2] and the geometric discord [4], it is a well accepted fact that the condition presented in Eq. (5) can be violated by certain two-qubit mixed states, see, for example, Refs. [51-54]. For higher dimensional system, entanglement quantified by Rényi entropies may also violate this condition [55]. But these violations are conceptually easy to understand as they are induced by the correlation measures defined from different perspectives. Here, as an unexpected result, we will show that even under the framework of weak measurements with different strengths [43], the resulting super discords similarly do not necessarily imply the same orderings of quantum states.

In the following, we illustrate the above argument through an explicit example. We consider a family of two-qubit states with maximally mixed marginals

$$
\rho_{A B}=\frac{1}{4}\left(I \otimes I+\sum_{i=1}^{3} c_{i} \sigma_{i} \otimes \sigma_{i}\right),
$$

where $I$ denotes the $2 \times 2$ identity operator, and $\sigma_{1,2,3}$ are the usual Pauli operators. Physical $\rho_{A B}$ are those with $\left(c_{1}, c_{2}, c_{3}\right)$ being confined to the tetrahedron with vertices $(-1,-1,-1)$, $(-1,1,1),(1,-1,1)$, and $(1,1,-1)$ [67]. These states are usually termed as the Bell-diagonal states as they can be decomposed into linear combinations of the four Bell states. The super discord for $\rho_{A B}$ is calculated as [50]

$$
\begin{aligned}
D_{w}^{\leftarrow}\left(\rho_{A B}\right)= & \frac{1-c_{1}-c_{2}-c_{3}}{4} \log _{2}\left(1-c_{1}-c_{2}-c_{3}\right) \\
& +\frac{1-c_{1}+c_{2}+c_{3}}{4} \log _{2}\left(1-c_{1}+c_{2}+c_{3}\right) \\
& +\frac{1+c_{1}-c_{2}+c_{3}}{4} \log _{2}\left(1+c_{1}-c_{2}+c_{3}\right) \\
& +\frac{1+c_{1}+c_{2}-c_{3}}{4} \log _{2}\left(1+c_{1}+c_{2}-c_{3}\right) \\
& -\frac{1-c \tanh x}{2} \log _{2}(1-c \tanh x) \\
& -\frac{1+c \tanh x}{2} \log _{2}(1+c \tanh x)
\end{aligned}
$$

where $c=\max \left\{\left|c_{1}\right|,\left|c_{2}\right|,\left|c_{3}\right|\right\}$.

In Fig. 1, we present an exemplified plot of the super discord as functions of $c_{3}$ for the Bell-diagonal states of Eq. (6) with $c_{1}=0.5$ and $c_{2}=-0.5$. The four curves from top to bottom are obtained by choosing the controlling parameters as $x=1.5,2.0,2.5$, and $\infty$ (corresponds to the normal discord), respectively, from which one can observe that there are states having different orderings induced by the super discord with the weak measurements of different strengths. This counterintuitive phenomenon can be further confirmed by the cyan (gray) shaded region shown in the inset of Fig. 1, which stands for the valid $\left(c_{1}, c_{2}\right)$ for which $\rho_{A B}$ of Eq. (6) with different $c_{3}$ can have different states ordering. It provides an intriguing perspective of the super discord in that it implies the quantum correlation in a state is not only measurement- 


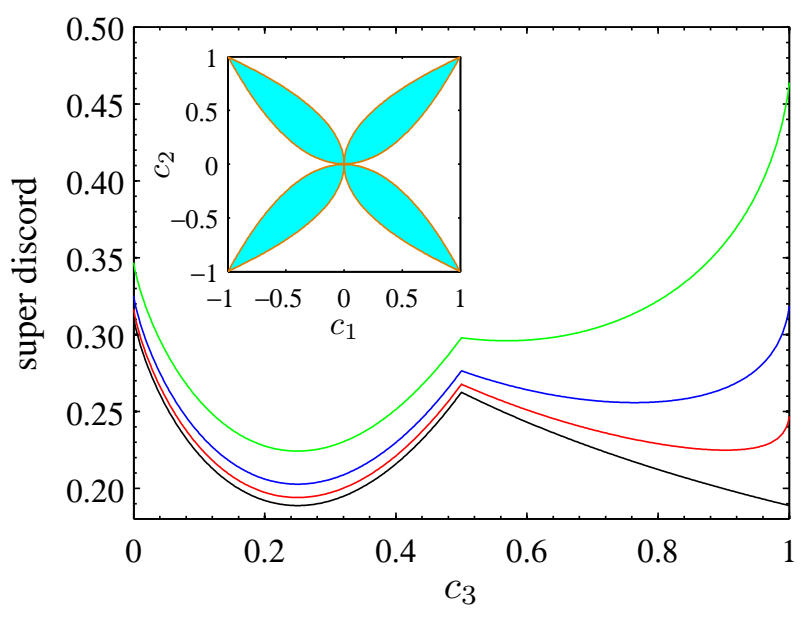

FIG. 1: (Color online) Super discord versus $c_{3}$ for the Bell-diagonal states of Eq. (6) with $c_{1}=0.5$ and $c_{2}=-0.5$. The green, blue, red, and black lines (from top to bottom) are plotted with $x=1.5,2.0$, 2.5 , and $\infty$ (i.e., the normal discord), respectively. The cyan (gray) shaded region in the inset represents valid $\left(c_{1}, c_{2}\right)$ for which the Belldiagonal states obtained by varying $c_{3}$ have different orderings induced by the super discord with different measurement strengths.

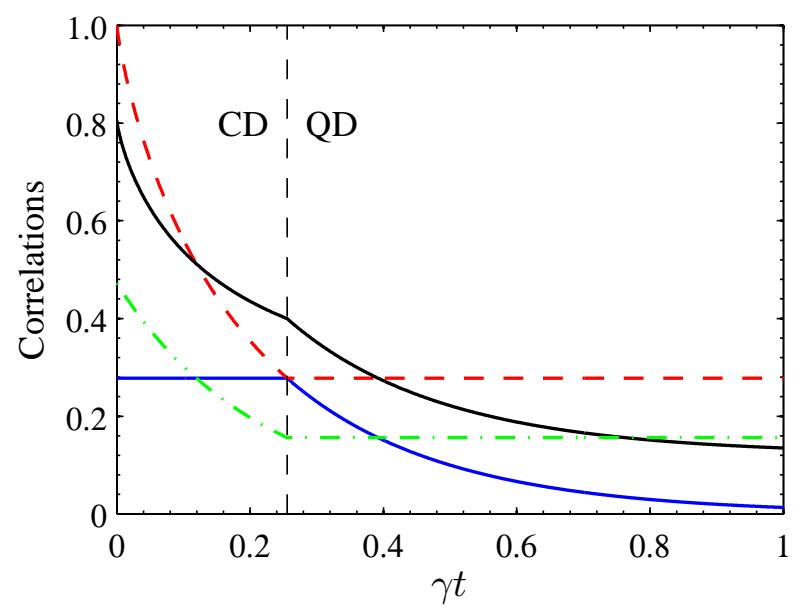

FIG. 2: (Color online) Normal discord [blue (gray) solid line], classical correlation (red dashed line), as well as the super discord (black solid line) and super classical correlation (green dash-dot line) obtained with $x=1.0$ versus $\gamma t$ under phase damping channel for the initial Bell-diagonal state of Eq. (6) with $c_{1}(0)=1, c_{2}(0)=-0.6$, and $c_{3}(0)=0.6$. CD and QD denote, respectively, the dynamical regimes of classical decoherence and quantum decoherence with respect to the projective measurement.

method-dependent [68, 69] but is also strongly dependent on the internal structures of the related measurements.

The lack of the unique states ordering may results in completely different dynamical behaviors of quantum correlations with respect to super discord. As an example, we consider the case of two qubits being prepared initially in the Belldiagonal state of Eq. (6), and subject to the same phase damping channels [64], the process of which preserves the Belldiagonal form of $\rho_{A B}$, with however the time dependence of the three parameters being given by $c_{1}(t)=c_{1}(0) \exp (-2 \gamma t)$, $c_{2}(t)=c_{2}(0) \exp (-2 \gamma t)$, and $c_{3}(t)=c_{3}(0)$, where $\gamma$ is the phase damping rate.

For this kind of local dissipative channel, it has been found that there are sudden transition from dynamical regimes of the classical decoherence (CD) to the quantum decoherence (QD) when considering the normal discord defined via the projective measurements [70], see, for example, the blue (gray) solid and red dashed lines shown in Fig. 2, When considering quantum and classical correlations based on the paradigm of weak measurements, however, the original two distinct regimes disappear. As can be seen from the black solid line shown in Fig. 2. the super discord decays with increasing $\gamma t$ even in the CD regime, which is in sharp contrast to that of the normal discord who is constant in time during the same regime.

Inspired by the connection between the normal discord and classical correlation [2], we further define the super classical correlation as

$$
C_{w}^{\leftarrow}\left(\rho_{A B}\right)=I\left(\rho_{A B}\right)-D_{w}^{\leftarrow}\left(\rho_{A B}\right)
$$

with $I\left(\rho_{A B}\right)=S\left(\rho_{A}\right)+S\left(\rho_{B}\right)-S\left(\rho_{A B}\right)$ being the quantum mutual information [64]. Then with the same parameters as those for the super discord in Fig. 2, we presented dynamics of $C_{w}^{\leftarrow}\left(\rho_{A B}\right)$ as the green dash-dot line in the same figure, from which one can note that it displays qualitatively similar behaviors as that for the normal classical correlation, i.e., it decays with time in the $\mathrm{CD}$ regime, and remains constant in the QD regime. We thus see that although the weak measurements can change the dynamical behaviors of super discord in the $\mathrm{CD}$ regime, it has no influence on the qualitative behaviors of the classical correlation. In fact, the unique ordering of states with the super classical correlations is universal for the class of Bell-diagonal states of Eq. (6), for which we always have

$$
\begin{aligned}
C_{w}^{\leftarrow}\left(\rho_{A B}\right)= & \frac{1-c \tanh x}{2} \log _{2}(1-c \tanh x) \\
& +\frac{1+c \tanh x}{2} \log _{2}(1+c \tanh x),
\end{aligned}
$$

which can be shown to be a monotonic increasing function of $c$ for arbitrary $x$, and therefore it always impose the same ordering for the Bell-diagonal states. But it should be note that the above argument does not hold for general case, as there are $\rho_{1}$ and $\rho_{2}$ such that the unique ordering condition is violated.

\section{MONOGAMY OF SUPER DISCORD}

We now turn to discuss the role weak measurements played in exploring monogamous character of quantum correlations. Due to the asymmetry of the super discord [48], there are two possible lines of research on this problem, which can be illustrated explicitly through the following two monogamy inequalities [56-63]

$$
\begin{aligned}
& D_{w}^{\leftarrow}\left(\rho_{A: B C}\right) \geqslant D_{w}^{\leftarrow}\left(\rho_{A B}\right)+D_{w}^{\leftarrow}\left(\rho_{A C}\right) \\
& D_{w}^{\rightarrow}\left(\rho_{A: B C}\right) \geqslant D_{w}^{\rightarrow}\left(\rho_{A B}\right)+D_{w}^{\vec{w}}\left(\rho_{A C}\right)
\end{aligned}
$$




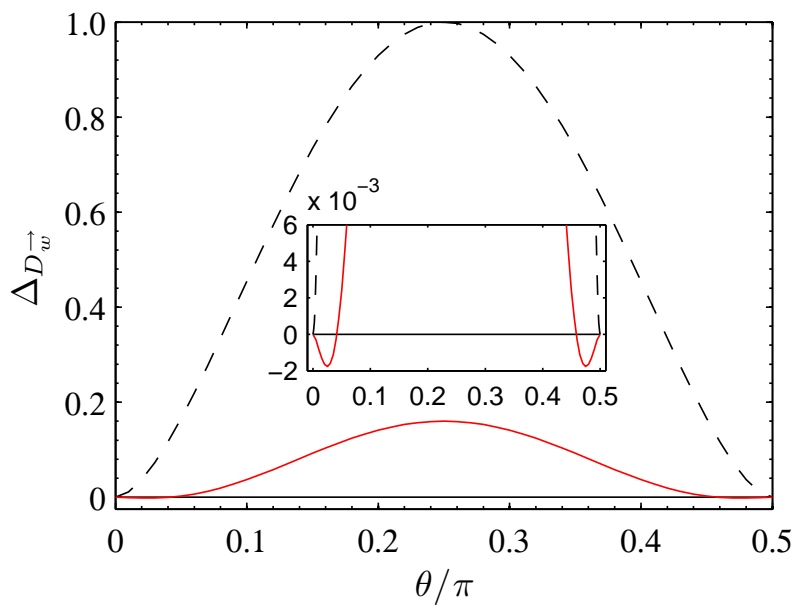

FIG. 3: (Color online) Dependence of the discord monogamy score $\Delta D_{w}$ on $\theta / \pi$ for $|\Psi\rangle_{\mathrm{GHZ}}$. The black dashed and the red solid lines are obtained with $x \rightarrow \infty$ and $x=0.5$, respectively. The inset is plotted for better visualizing violation of the monogamy condition during the small and large $\theta / \pi$ regions.

where the first one is formulated with the measurements being performed on different subsystems $B, C$ and $B C$ of $\rho_{A B C}$ [56-62], and the second one is formulated with the measurements being performed on the same subsystem $A$ [63]. For convenience of later presentation, we further define $\Delta D_{w}^{\alpha}=$ $D_{w}^{\alpha}\left(\rho_{A: B C}\right)-D_{w}^{\alpha}\left(\rho_{A B}\right)-D_{w}^{\alpha}\left(\rho_{A C}\right)(\alpha \in\{\leftarrow, \rightarrow\})$ as the related discord monogamy score [60].

As the super discord is an extension of the normal discord, and the normal discord has been found to be monogamous for certain classes of quantum states (e.g., the generalized GHZclass state) [57], it is natural to ask whether this monogamy nature is universal for the super discord with arbitrary measurement strengths, or whether the super discord still respect monogamy for these states?

Here, we will show through some explicit examples the answer to this question is indeed state-dependent, that is to say, these states can be monogamous as well as polygamous with respect to super discord. Our first exemplification is that of the generalized GHZ states

$$
|\Psi\rangle_{\mathrm{GHZ}}=\cos \theta|000\rangle+\sin \theta|111\rangle,
$$

which is known to be monogamous with respect to the normal discord with infinite measurement strength $x$ [57]. When considering the super discord defined with finite $x$, the two monogamy conditions in Eq. (10) are in fact equivalent due to the exchange symmetry of $|\Psi\rangle_{\text {GHZ }}$. In Fig. 3 we plotted the related monogamy score $\Delta D_{w}^{\rightarrow}$ against $\theta / \pi$ with the measurement strengths $x \rightarrow \infty$ (corresponds to the normal discord) and $x=0.5$, respectively. The states are monogamous whenever $\Delta D_{w}$ take positive values. This figure shows evident transitions from observation to violation of monogamy for the super discord. More specifically, the super discord does not respect monogamy during the small and large regions of $\theta / \pi$. This result is interesting as it implies that the monogamy property of discord, even for those defined under the same formal-
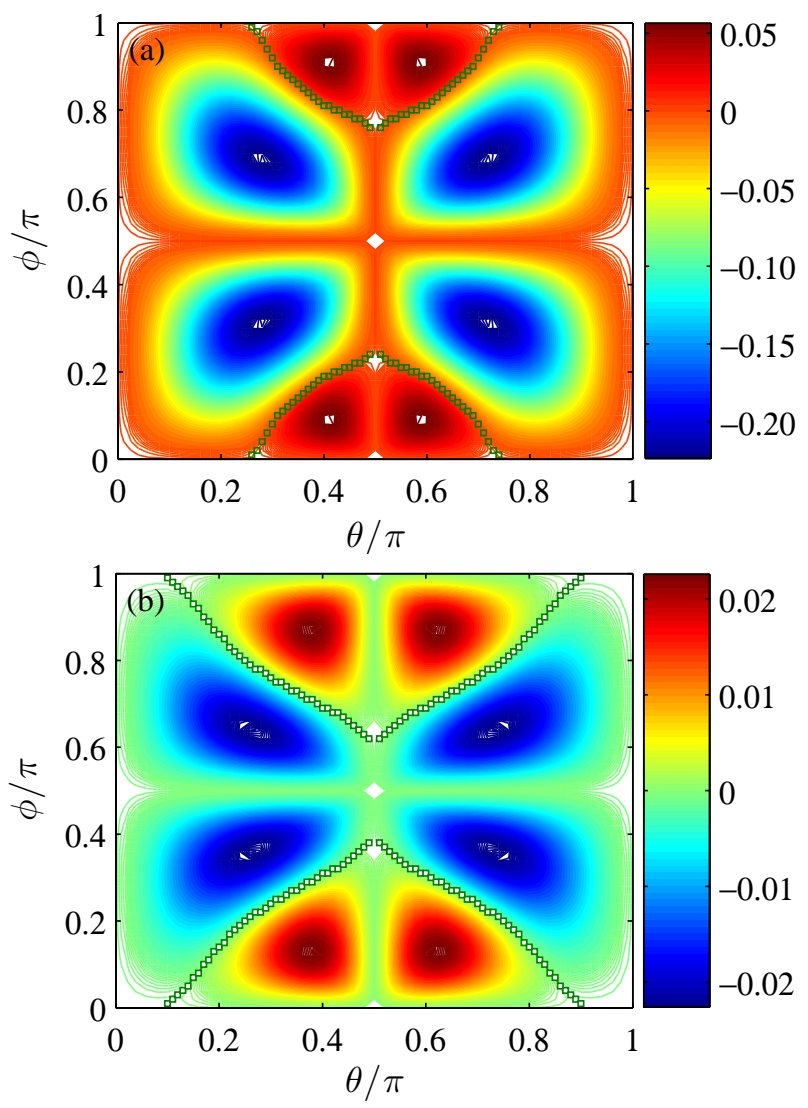

FIG. 4: (Color online) Contour plots of the discord monogamy score $\Delta D_{w}$ against $\theta / \pi$ and $\phi / \pi$ for $|\Psi\rangle_{W}$ with $x \rightarrow \infty$ (a) and $x=0.5$ (b), respectively. The regions up and below the green hollow squares represent valid $|\Psi\rangle_{W}$ with monogamy.

ism of measurements, is not only state-dependent but is also determined by the intrinsic properties, e.g., the measurement strengths, of the related measurements.

As another example, we consider the generalized $W$-class states

$|\Psi\rangle_{W}=\sin \theta \cos \phi|011\rangle+\sin \theta \sin \phi|101\rangle+\cos \theta|110\rangle$,

for which the two monogamy inequalities in Eq. (10) are no longer equivalent, and when evaluated via the normal discord, the first one is always violated [57], while the second one may be satisfied or violated [63].

When considering quantum correlations captured by the super discord, our results revealed that the first monogamy condition in Eq. (10) still remains violated for the weak measurements of arbitrary strengths. But if we consider the second condition, the case will be very different. As can be seen from the contour plots shown in Fig. 4, the monogamy property turns out to be state-dependent, and in contrast to that for the generalized GHZ-class states, here the $(\theta, \phi)$ regions for monogamy are enlarged with finite measurement strength.

From a practical point of view, the monogamous nature of the normal discord can be used to distinguish two stochas- 


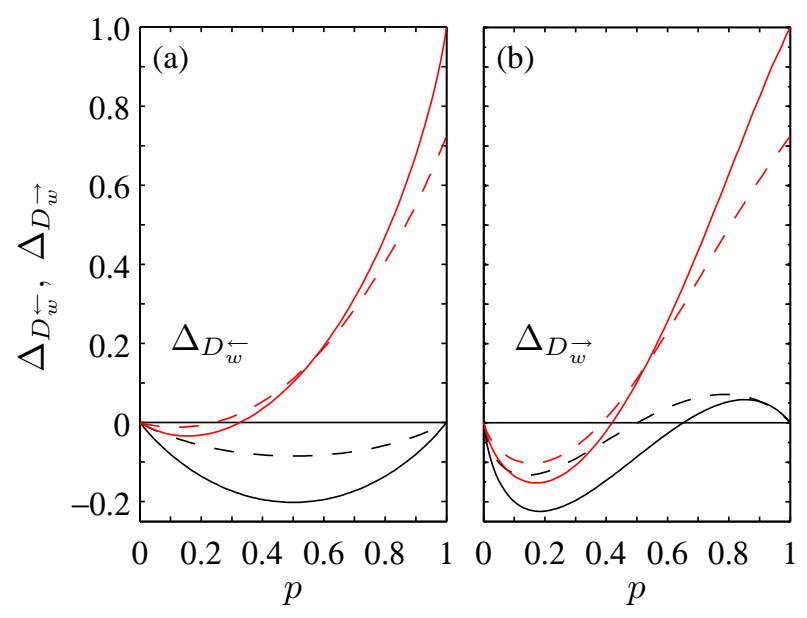

FIG. 5: (Color online) Dependence of the discord monogamy score $\Delta D_{w}^{\leftarrow}$ and $\Delta D_{w}^{\rightarrow}$ on $p$ for $|\psi(p, \epsilon)\rangle$. The black and red (gray) solid lines are plotted for the normal discord with $\epsilon=1$ and $\epsilon=0.5$, respectively; while the black and red (gray) dashed lines are plotted for the super discord with $x=1.5$, and $\epsilon=1$ and $\epsilon=0.5$, respectively.

tic local operations and classical communication (SLOCC) inequivalent classes of tripartite states, i.e., the generalized GHZ and $W$ classes [57]. But when evaluated via the super discord, the above results revealed that this application will does not applicable, as the GHZ-class states can also be monogamous as well as polygamous for this case (see, Fig. 3). This exhibits another perspective of the weak measurements in defining quantum correlations, which is beyond our expectation.

Finally, we remark that the change to the monogamy nature of quantum states with respect to the super discord can also happen when the weak measurements are performed on different subsystems. See, for example, the results displayed in Fig. 5. which is plotted for the tripartite pure states $|\psi(p, \epsilon)\rangle=$ $\sqrt{p \epsilon}|000\rangle+\sqrt{p(1-\epsilon)}|111\rangle+\sqrt{(1-p) / 2}(|101\rangle+|110\rangle)$.

\section{CONCLUSION}

Weak measurements are important complementary to the standard measurement in quantum theory. By reconsidering the processes of weak measurements with different strengths, we showed that while being able to capture more quantum correlation, it can also induce other counterintuitive effects meanwhile. As the first exemplification, we showed that the weak measurements with different strengths can impose different orderings of quantum states. This effect is very different from those observed with different quantum correlation measures, e.g., the entropic and the geometric measures of the normal discord, and it may results in unexpected dynamical behaviors of quantum correlations. Moreover, we have also showed that the monogamous nature of the normal discord for certain classes of quantum states can be changed by the weak-measurement-defined super discord, and this change can even invalidate the feasibility of some quantum tasks, such as the detection of two SLOCC-inequivalent classes of tripartite states based on monogamy [57]. In view of these facts, we then conclude that the weak measurements play a dual role in defining quantum correlations.

On the other hand, since different physical systems may naturally interact strongly or weakly with probing systems, the full description of measurement-dependent quantum correlation may be complete only when weak measurement with adjustable strengths are considered. This may also provide a full quantification of quantum correlation restricted experimentally to some specified quantum systems. In particular, in case the quantum correlation based on weak measurements may enhance or diminish its usefulness in some protocols, a complete view of super quantum discord is necessary and may shed light on our understanding of other quantum characteristics.

\section{ACKNOWLEDGMENTS}

This work was supported by NSFC (11205121, 10974247, 11175248), the "973" program (2010CB922904), NSF of Shaanxi Province (2010JM1011), and the Scientific Research Program of the Education Department of Shaanxi Provincial Government (12JK0986).
[1] Horodecki, R., Horodecki, P., Horodecki, M., Horodecki, K.: Rev. Mod. Phys. 81, 865 (2009)

[2] Ollivier, H., Zurek, W.H.: Phys. Rev. Lett. 88, 017901 (2001)

[3] Henderson, L., Vedral, V.: J. Phys. A 34, 6899 (2001)

[4] Dakić, B., Vedral, V., Brukner, Č.: Phys. Rev. Lett. 105, 190502 (2010)

[5] Modi, K., Paterek, T., Son, W., Vedral, V., Williamson, M.: Phys. Rev. Lett. 104, 080501 (2010)

[6] Datta, A., Shaji, A., Caves, C.M.: Phys. Rev. Lett. 100, 050502 (2008)

[7] Werlang, T., Trippe, C., Ribeiro, G.A.P., Rigolin, G.: Phys. Rev. Lett. 105, 095702 (2010)
[8] Werlang, T., Rigolin, G.: Phys. Rev. A 81, 044101 (2010)

[9] Li, Y.C., Lin, H.Q.: Phys. Rev. A 83, 052323 (2011)

[10] Dakić, B., Lipp, Y.O., et al.: Nat. Phys. 8, 666 (2012)

[11] Roa, L., Retamal, J.C., Alid-Vaccarezza, M.: Phys. Rev. Lett. 107, 080401 (2011)

[12] Li, B., Fei, S.M., Wang, Z.X., Fan, H.: Phys. Rev. A 85, 022328 (2012)

[13] Datta, A., Gharibian, S.: Phys. Rev. A 79, 042325 (2009)

[14] Wu, S., Poulsen, U.V., Mølmer, K.: Phys. Rev. A 80, 032319 (2009)

[15] Gu, M., Chrzanowski, H.M., et al.: Nat. Phys. 8, 671 (2012)

[16] Madhok, V., Datta, A.: Phys. Rev. A 83, 032323 (2011) 
[17] Cavalcanti, D., Aolita, L., Boixo, S., Modi, K., Piani, M., Winter, A.: Phys. Rev. A 83, 032324 (2011)

[18] Adhikari, S., Banerjee, S.: Phys. Rev. A 86, 062313 (2012)

[19] Streltsov, A., Kampermann, H., Bruß, D.: Phys. Rev. Lett. 107, 170502 (2011)

[20] Hu, X., Fan, H., Zhou, D.L., Liu, W.M.: Phys. Rev. A 85, 032102 (2012)

[21] Hu, X., Fan, H., Zhou, D.L., Liu, W.M.: Phys. Rev. A 87, 032340 (2013)

[22] Gessner, M., Laine, E.M., Breuer, H.P., Piilo, J.: Phys. Rev. A 85, 052122 (2012)

[23] Abad, T., Karimipour, V., Memarzadeh, L.: Phys. Rev. A 86, 062316 (2012)

[24] Galve, F., Plastina, F., Paris, M.G.A., Zambrini, R.: Phys. Rev. Lett. 110, 010501 (2013)

[25] Yu, X.Y., Li, J.H., Li, X.B.: Sci. China Phys. Mech. 55, 815 (2012)

[26] Yue, H.D., Zhang, Y., Gong J.: Sci. China Phys. Mech. 55, 1641 (2012)

[27] Zhu, Y.Y., Zhang, Y.: Sci. China Phys. Mech. 55, 2081 (2012)

[28] Qian, Y., Zhang, Y.Q., Xu, J.B.: Chinese Sci. Bull. 57, 1637 (2012)

[29] Ma, X.S., Qiao, Y., Zhao, G.X., Wang, A.M.: Sci. China Phys. Mech. 56, 600 (2013)

[30] Modi, K., Brodutch, A., Cable, H., Paterek, T., Vedral, V.: Rev. Mod. Phys. 84, 1655 (2012)

[31] Giorgi, G.L., Bellomo, B., Galve, F., Zambrini, R.: Phys. Rev. Lett. 107, 190501 (2011)

[32] Luo, S., Fu, S.: Phys. Rev. Lett. 106, 120401 (2011)

[33] Luo, S.: Phys. Rev. A 77, 022301 (2008)

[34] Bellomo, B., Giorgi, G.L., et al.: Phys. Rev. A 85, 032104 (2012)

[35] Aaronson, B., Franco, R.L., Compagno, G., Adesso, G.: New J. Phys. 15, 093022 (2013)

[36] Zhou, T., Cui,J., Long, G.L.: Phys. Rev. A 84, 062105 (2011)

[37] Hamieh, S., Kobes, R., Zaraket, H.: Phys. Rev. A 70, 052325 (2004)

[38] Galve, F., Giorgi, G.L., Zambrini, R.: Europhys. Lett. 96, 40005 (2011)

[39] Ritchie, N.W.M., Story, J.G., Hulet, R.G.: Phys. Rev. Lett. 66, 1107 (1991)

[40] Pryde, G.J., O’Brien, J.L., et al.: Phys. Rev. Lett. 94, 220405 (2005)

[41] Hosten, O., Kwiat, P.: Science 319, 787 (2008)

[42] Wu, S.J.: Sci. Rep. 3, 1193 (2013)

[43] Oreshkov, O., Brun, T.A.: Phys. Rev. Lett. 95, 110409 (2005)
[44] Aharonov, Y., Albert, D.Z., Vaidman, L.: Phys. Rev. Lett. 60, 1351 (1988)

[45] Korotkov, A.N., Jordan, A.N.: Phys. Rev. Lett. 97, 166805 (2006)

[46] Sun, Q., Al-Amri, M., Zubairy, M.S.: Phys. Rev. A 80, 033838 (2009)

[47] Wu, S., Żukowski, M.: Phys. Rev. Lett. 108, 080403 (2012)

[48] Singh, U., Pati, A.K.: Ann. Phys. (NY) 343, 141 (2014)

[49] Li, B., Chen, L., Fan, H.: Phys. Lett. A, doi:10.1016/j.physleta.2014.02.036

[50] Wang, Y.K., Ma, T., Fan, H., Fei, S.M., Wang, Z.X.: Quantum Inf. Process. 13, 283 (2014)

[51] Miranowica, A.: Phys. Lett. A 327, 272 (2004)

[52] Yeo, Y., An, J.-H., Oh, C.H.: Phys. Rev. A 82, 032340 (2010)

[53] Hu, M.L., Fan, H.: Ann. Phys. (NY) 327, 851 (2012)

[54] Okras, M., Walczak, Z.: Europhys. Lett. 98, 40003 (2012)

[55] Cui, J., Gu, M., Kwek, L.C., Santos, M.F., Fan, H., Vedral, V.: Nat. Commun. 3, 812 (2012)

[56] Giorgi, G.L.: Phys. Rev. A 84, 054301 (2011)

[57] Prabhu, R., Pati, A.K., Sen(De), A., Sen, U.: Phys. Rev. A 85, 040102 (2012)

[58] Sudha, Usha Devi, A.R., Rajagopal, A.K.: Phys. Rev. A 85, 012103 (2012)

[59] Streltsov, A., Adesso, G., Piani, M., Bruß, D.: Phys. Rev. Lett. 109, 050503 (2012)

[60] Bera, M.N., Prabhu, R., Sen(De), A., Sen, U.: Phys. Rev. A 86, 012319 (2012)

[61] Braga, H.C., Rulli, C.C., de Oliveira ,T.R., Sarandy M.S.: Phys. Rev. A 86, 062106 (2012)

[62] Fanchini, F.F., de Oliveira, M.C., Castelano, L.K., Cornelio, M.F.: Phys. Rev. A 87, 032317 (2013)

[63] Ren, X.J., Fan, H.: Quantum Inf. Comput. 13, 0469 (2013)

[64] Nielsen, M.A., Chuang, I.L.: Quantum Computation and Quantum Information. Cambridge University Press, Cambridge (2000)

[65] Wootters, W.K.: Phys. Rev. Lett. 80, 2245 (1998)

[66] Vidal, G., Werner, R.F.: Phys. Rev. A 65, 032314 (2002)

[67] Horodecki, R., Horodecki, M.: Phys. Rev. A 54, 1838 (1996)

[68] D'Espagnat, B.: Veiled Reality: An Analysis of Present-Day Quantum Mechanical Concepts, Westview Press (2003)

[69] Long, G.L., Zhou, Y.F., Jin, J.Q., Sun, Y., Lee, H.W.: Found. Phys. 36, 1217 (2006)

[70] Mazzola, L., Piilo, J., Maniscalco, S.: Phys. Rev. Lett. 104, 200401 (2010) 